\title{
Identity of the universal repulsive-core singularity with Yang-Lee edge criticality
}

\author{
Youngah Park ${ }^{1,2}$ and Michael E. Fisher ${ }^{1}$ \\ ${ }^{1}$ Institute of Physical Science and Technology, University of Maryland. College Park, MD 20742 \\ ${ }^{2}$ Department of Physics, Myong Ji University, Yongin, Kyonggi-do, 449-728, Korea
}

(July 1999)

\begin{abstract}
Lattice and continuum fluid models with repulsive-core interactions typically display a dominant, critical-type singularity on the real, negative activity axis. Lai and Fisher recently suggested, mainly on numerical grounds, that this repulsive-core singularity is universal and in the same class as the Yang-Lee edge singularities, which arise above criticality at complex activities with positive real part. A general analytic demonstration of this identification is presented here using a field-theory approach with separate representation of the repulsive and attractive parts of the pair interactions.
\end{abstract}

\section{INTRODUCTION}

In 1952 Lee and Yang [1] proved that the zeroes of the partition function of a ferromagnetic Ising model (or equivalently, a lattice gas with single-site hard cores) as a function of complex magnetic field $H$ (or chemical potential $\mu$ ) are confined to the imaginary $H$ axis for real temperatures $T$. Yang and Lee argued [2] that for a system above its critical temperature $T_{c}$, the partition function must be nonzero throughout some neighborhood of the real axis in the complex reduced magnetic-field plane,

$$
h=H / k_{B} T \equiv h^{\prime}+i h^{\prime \prime} .
$$

Thus for $T>T_{c}$, a gap free of zeros will be found on the imaginary $h$ axis with edges at, say, $\pm i h_{\sigma}(T)$. Equivalently, in fluid-language, for $T>T_{c}$, there will be a gap in the complex activity plane with edges at

$$
z=z_{\sigma}^{\prime}(T) \pm i z_{\sigma}^{\prime \prime}(T)
$$

as illustrated in Fig. 1. Here we define the reduced activity in $d$ spatial dimensions by

$$
z=e^{\beta \mu} v_{0} / \Lambda_{T}^{d}
$$

where $\beta=1 / k_{B} T$, while $v_{0}$ is a microscopic reference volume (taken as the cell volume for a lattice gas) and $\Lambda_{T}$ is the thermal de Broglie wavelength.

If one defines the density of Yang-Lee zeros, $g\left(h^{\prime \prime}\right)$, so that when $N$, the number of spins (or lattice sites) becomes infinite, $N g\left(h^{\prime \prime}\right) d h^{\prime \prime}$ approaches the number of zeros between $i h^{\prime \prime}$ and $i\left(h^{\prime \prime}+d h^{\prime \prime}\right)$ on the imaginary $h$ axis, one must have $g\left(h^{\prime \prime}\right) \equiv 0$ for $\left|h^{\prime \prime}\right|<$ $h_{\sigma}(T)$, for $T>T_{c}$. Kortman and Griffiths [3] pointed out that the density of zeros beyond the gap should be expected to exhibit a power law singularitythe Yang-Lee edge singularity 泊—of the form

$$
g\left(h^{\prime \prime}\right) \sim|| h^{\prime \prime}\left|-h_{\sigma}(T)\right|^{\sigma} \quad \text { for } \quad\left|h^{\prime \prime}\right| \rightarrow h_{\sigma}(T)-.
$$

This singularity proves analogous to an ordinary critical point obeying scaling laws and exponent relations although there is only one relevant scaling field [3, 4].
Thus the basic exponent $\sigma$ in (4) satisfies $\sigma=1 / \delta=$ $(d-2+\eta) /(d+2-\eta)$ [4].

In a general renormalization group analysis, it was shown [4] that the field theory controlling the Yang-Lee fixed point is described by a pure imaginary $i \varphi^{3}$ coupling. This leads to a critical dimension $d_{c}=6$ above which the classical, mean-field value [4] $\sigma=\frac{1}{2}$ applies. To first order in $\epsilon=6-d>0$ one has $\sigma(d) \stackrel{1}{=} \frac{1}{2}-\frac{1}{12} \epsilon$ and $\eta=-\frac{1}{9} \epsilon$. Expansions of $\sigma(d)$ to order $\epsilon^{3}$ are known [5] and one has $\sigma(1)=-\frac{1}{2} \sqrt{6}$ ], $\sigma(2)=-\frac{1}{6}$, and finds, numerically, $\sigma(3) \simeq 0.088$ [7] : see Lai and Fisher [7] who review previously known relationships of the YangLee edge singularity to a number of different problems, specifically, isotropic branched polymers or, equivalently, undirected lattice animals with or without loops allowed, in $(d+2)$ dimensions $[8,9]$; Anderson localization [10]; and directed branched polymers (or directed, loop-free lattice animals) in $d+1$ dimensions [11].

By contrast, an apparently quite different type of singularity arises in fluid systems when the particle interactions have repulsive cores. If the pair interaction potentials are purely repulsive (i.e., positive), the (reduced) cluster integrals, $b_{n}(T)$, in the activity or fugacity series for the (reduced) pressure, namely,

$$
\bar{p} \equiv \frac{p v_{0}}{k_{B} T}=\sum_{n=1}^{\infty} b_{n}(T) z^{n},
$$

are known 12 to alternate in sign: this implies a dominant singularity on the negative $z$-axis that determines the radius of convergence, $R$, of the series: see Fig. 1 . In the vicinity of this singularity, say at $z=z_{0}(T)=-R$, the reduced pressure can be written as

$$
\begin{aligned}
\bar{p}(z)= & \bar{p}_{0}+\bar{p}_{1}\left(z-z_{0}\right)+\bar{p}_{2}\left(z-z_{0}\right)^{2}+\cdots \\
& +P\left(z-z_{0}\right)^{\phi}\left[1+a_{\theta}\left(z-z_{0}\right)^{\theta}+\cdots\right]+\cdots
\end{aligned}
$$

where the exponents $\phi$ and $\theta$ are anticipated to be nonintegral.

Indeed, in 1984, Poland 13 studied a variety of lattice models and a continuum fluid of hard squares and 
proposed that this repulsive-core singularity is characterized by a universal exponent $\phi(d)$. Subsequent confirmation came from Baram and Luban [14] who investigated further models including dimers on lattices, parallel hypercubes in continuum space and the soft-core single-component Gaussian-molecule model. More recently, Lai and Fisher [7] found similar behavior in a binary Gaussian-molecule mixture using very long series expansions for $d=1, \cdots, 6$. In that case the singularity at $z=z_{0}$ was drawn out into a continuous locus; but the estimates for $\phi(d)$ supported the universality hypothesis. (Precise estimates, for the leading "correction-toscaling" exponent $\theta(d)$, in (6) for all $d$, including $\theta(1)=1$, $\theta(2)=\frac{5}{6}$, and $\theta(3) \simeq 0.62$, were also generated [7].)

However, Lai and Fisher [7] noticed in particular that, when compared with previous knowledge about the Yang-Lee edge exponent $\sigma(d)$, the exact results for $\phi(d)$ for $d=1$ and $d=\infty$ [15] and for $d=2$ [14,16], and the various numerical estimates [7] for $d \geq 2$, strongly suggested the identification

$$
\phi(d)=\sigma(d)+1 \quad \text { for } \quad \text { all } \quad d .
$$

Hence they proposed [7] that the universal repulsive-core singularity belongs to the Yang-Lee edge critical universality class.

In analytical support of this identification (for general d) they appealed to earlier work by Kurtze and Fisher 17] who had proved that when $T \rightarrow \infty$ the Yang-Lee edge singularity in ferromagnetic Ising models precisely describes the dominant singularity on the negative $z$ axis of a fluid of hard dimers on the same lattice, each dimer occupying one bond and two adjacent lattice sites. This correspondence with a dimer gas was carried further by Shapir [18] who used field-theoretic arguments to demonstrate the identity generically for all $T>T_{c}$. However, dimers are rather special objects with orientational degrees of freedom (although these seem, post facto, to have no effect on $\phi(d)$ 14). Furthermore, Shapir's fieldtheoretic approach was rather special and did not seem extendable to more general particles with soft repulsive cores, with the additional presence of attractive forces, or to systems also displaying critical behavior for positive $z$ [7] (as illustrated in Fig. 1).

In this article we repair this gap in the theory. Specifically, we consider a general single-component fluid with a pair interaction potential, $U(\mathbf{r})$, which contains both repulsive and attractive parts: of course, repulsive terms are always essential to ensure thermodynamic stability. To be concrete and explicit we analyze lattice systems in which multiple occupancy of a site is forbidden; however, it must be stressed that the repulsive interactions we consider are not confined to such trivial single-site hard cores. On the contrary, essentially we suppose only that the potential $U(\mathbf{r})$ is of finite range and may be decomposed according to

$$
\hat{U}(\mathbf{k})=\sum_{\mathbf{R} \neq 0} e^{i \mathbf{k} \cdot \mathbf{R}} U(\mathbf{R})=\hat{w}(\mathbf{k})-\hat{v}(\mathbf{k}),
$$

where the sum runs over lattice sites $\mathbf{R}=\mathbf{r}_{j}$ while the repulsive and attractive parts, $\hat{w}(\mathbf{k})$ and $\hat{v}(\mathbf{k})$, respectively, are both positive (if they do not vanish identically). More specifically we will use

$$
\begin{aligned}
& \hat{w}(\mathbf{k})=\hat{w}_{0}\left(1-k^{2} a^{2}+\cdots\right)>0, \\
& \hat{v}(\mathbf{k})=\hat{v}_{0}\left(1-k^{2} b^{2}+\cdots\right)>0,
\end{aligned}
$$

so that $a$ and $b$ represent the interaction ranges of the repulsive and attractive components. The real-space potentials acting between sites $j$ and $k(j \neq k)$, namely, $w_{j k}=w\left(\mathbf{r}_{k}-\mathbf{r}_{j}\right)$ and $v_{j k}=v\left(\mathbf{r}_{k}-\mathbf{r}_{j}\right)$, follow by Fourier inversion. For $d=3$ one may imagine Yukawa forms: $w(\mathbf{r})=W_{0} e^{-r / a} / r$ and $v(\mathbf{r})=V_{0} e^{-r / b} / r$ with $a<b$; but that is certainly not essential. Likewise, the leading lattice isotropy assumed for convenience in (9) and (10) is not necessary. Our treatment extends straightforwardly to multicomponent fluids [7] and, at least formally, generalizes readily to continuum systems.

On the basis of (8)-(10), we develop a field-theoretic analysis and show that there is, in general, a repulsivecore singularity at some $z_{0}(T)$ on the negative activity axis (see Fig. 1) that is described within an LGW renormalization group framework by a fixed point Hamiltonian with a purely imaginary cubic coupling, $i \varphi^{3}$, and, hence, lies in the same universality class as Yang-Lee edge criticality. A more or less novel aspect of our treatment is that separate - sine-Gordan [19] and Kac-HubbardStratonovich (KHS) 20 22]-transformations are used for handling the repulsive and attractive parts of the interaction potential: compare with Refs. 23 and 24 .

\section{FORMULATION}

For a lattice of volume $V$ with sites labeled $j, k, l=$ $1,2, \cdots, \mathcal{N}=V / v_{0}$, let $\rho_{j}=0$ or 1 according as site $j$ is or is not occupied by a particle. Then, recalling (3) and (8)-(10) et seq., the grand partition function for the lattice system is

$$
\mathcal{Z}(T, z)=\operatorname{Tr}_{\rho}^{\mathcal{N}}\left\{z^{\sum_{j} \rho_{j}} \exp \left[-\frac{1}{2} \beta \sum_{j \neq k} \rho_{j}\left(w_{j k}-v_{j k}\right) \rho_{k}\right]\right\} .
$$

Now, utilizing the positivity of $\hat{w}(\mathbf{k})$ and $\hat{v}(\mathbf{k})$, we may apply a sine-Gordon transformation [19 to the repulsive terms, $w_{j k}$, and a KHS transformation [20 22] to the attractive terms $v_{j k}$ (where, as usual, it is most convenient to utilize periodic lattice boundary conditions). Neglecting an unimportant constant factor, this yields 


$$
\mathcal{Z}=\int \frac{\mathcal{D} \varphi}{\sqrt{|\mathbf{w}|}} \int \frac{\mathcal{D} \chi}{\sqrt{|\mathbf{v}|}} \exp \left[-\frac{1}{2} \sum_{j, k} \varphi_{j} w_{j k}^{-1} \varphi_{k}-\frac{1}{2} \sum_{l, m} \chi_{l} v_{l m}^{-1} \chi_{m}\right] \text { III. TRIVIAL HARD CORES AND GAS-LIQUID }
$$

$$
\times \operatorname{Tr}_{\rho}^{\mathcal{N}}\left\{z^{\sum_{j} \rho_{j}} \exp \left[\sum_{j}\left(-i \varphi_{j}+\chi_{j}\right) \rho_{j}\right]\right\}
$$

(12) We remark, first, that following the pioneering study of Hubbard and Schofield [26], all authors interested in ordinary gas-liquid criticality have treated the repulsive interactions in a fluid by use of a reference system: for a recent example, see Brilliantov [27]. Unless this reference fluid is essentially trivial, as for single-site hard cores on a lattice, this entails increasingly detailed knowledge of the correlation functions of the repulsive-core system [26,27. Nevertheless, it will be helpful for us to make contact with this approach by, initially, neglecting the nontrivial repulsive interactions embodied in $w(\mathbf{r})$. If the attractions are also neglected the grand partition function (11) reduces simply to $\mathcal{Z}=(1+z)^{\mathcal{N}}$. This predicts a repulsive-core singularity at $z_{0}=-1$ (all $T$ ) with an exponent $\phi(d=0)=0(\log )[7]$. Evidently, the actual dimensionality, $d$, plays no role.

Now, following traditional treatments (e.g., [25]) let us introduce attractive terms with $\hat{v}(\mathbf{k})>0$. The KHS transformation then leads to the saddle-point equation (18) in which, now, $U_{0}=-\hat{v}_{0}<0$ is negative and we can identify $\psi$ directly with $\chi_{0}$ (since $\hat{w}_{0}=0$ and $\int \mathcal{D} \varphi$ etc., can be ignored). Fig. 2 then provides a graphical representation of (18) which can be readily analyzed: note that the bold curve depicts $\psi e^{-\psi}$ which has a point of inflection at $\psi_{c}=2$ and a corresponding tangent that intersects the axis at $\psi=4$ : see the dashed line.

By inspection, one then sees that when $z$ increases from $z=0$ (along the real axis) for temperatures such that $\beta \hat{v}_{0}<4$ there is always a single saddle-point solution, $\psi_{0}(T, z)$, which varies analytically with $T$ and $z$ : see, e.g., the lines labelled (a) and (b) in Fig. 2. Conversely, for lower $T$, when $\beta \hat{v}_{0}>4$, there is a single analytic solution, $\psi_{0}(T, z)$, for small $z$ [as on the line (c)] but for an intermediate range of $z$ three distinct solutions arise, as illustrated by (d); finally, for larger $z$ only a single solution remains: line (e). Evidently the three solutions merge at a bifurcation point determined by the inflection point at $\psi_{c}=2$ : this leads to $\beta_{c} \hat{v}_{0}=4$ and $z_{c}=e^{-2} \simeq 0.135$. Expanding $\mathcal{H}[\chi]$ in powers of $\left(\beta-\beta_{c}\right),\left(z-z_{c}\right)$ and $\delta \chi_{j}=\chi_{j}-\chi_{c}$, all taken as real variables, shows that this saddle-point bifurcation simply represents the anticipated classical or mean-field gas-liquid critical point at $k_{B} T_{c}^{0}=\frac{1}{4} \hat{v}_{0}$. As usual, the corresponding LGW Hamiltonian can be used as a starting point in a field-theoretic renormalization-group (RG) treatment which then leads to all the standard results.

On the other hand, for $\beta \lesssim \beta_{c}$, i.e., $T \gtrsim T_{c}$, one can follow Ref. 泊 and discover two Yang-Lee edge singularities at complex $z$ (with small imaginary parts when $T$ is near $T_{c}$ ): at the saddle-point level these have $\sigma=\frac{1}{2}$; but the fixed-point Hamiltonian is controlled by an $i(\delta \chi)^{3}$ coupling leading, as explained above, to an $\mathrm{RG} \epsilon=6-d$ expansion 偖.

If, next, small repulsive terms, $\hat{w}>0$, are introduced,
Furthermore, by substitution of any solution of (18) on the right hand sides of (15) and (16) one obtains the separate solutions $\varphi_{0}$, which may evidently be imaginary, and $\chi_{0}$. 
the overall interaction parameter $U_{0}=\hat{w}_{0}-\hat{v}_{0}$, in (18) remains negative and the arguments proceed in essentially the same manner (although, in due course, the field $\varphi$ for the repulsive terms would normally be integrated out). As expected, neither the usual gas-liquid nor the YangLee edge singularities undergo any change in character. However, if $\hat{w}_{0}$ becomes sufficiently large, $U_{0}$ becomes positive and then, clearly, the previous analysis fails! In particular, as seen in Fig. 3 [lines (a) and (b)], for positive (real) $z$, there is always only a single, smoothly varying saddle-point solution, $\psi_{0}(T, z)$. Of course, this does not (necessarily) mean that the usual gas-liquid and YangLee edge singularities are lost. Rather, the form of the Hamiltonian, $\mathcal{H}[\varphi, \chi]$, represents an inadequate starting point for a perturbative RG approach: instead, it becomes necessary to integrate out (at least to some degree) the repulsive terms, i.e., to perform some $\int \mathcal{D} \varphi$ integrals and, thereby, make contact with the reference-fluid treatments 2527 .

\section{THE REPULSIVE-CORE SINGULARITY}

On the other hand, when the repulsions dominate, so that $U_{0}>0$ (as we will assume hereon), one sees from Fig. 3 [e.g., lines (c) and (d)] that for a real negative $z$ there is a unique positive saddle-point solution, say $\psi_{-}(T, z)<1$, that vanishes when $z \rightarrow 0$. However, when $z$ approaches $z_{0}(T)<0$ (from above) a bifurcation point is reached at which the saddle-point must become complex: see the broken line in Fig. 3 that corresponds to $z=z_{0}(T)$. (At $z=z_{0}$ a second, larger saddle-point solution, $\psi_{+}(T, z)$, merges with $\psi_{-}(T, z)$. For $z$ much larger, additional real negative saddle-point solutions, $\psi_{ \pm}^{\prime}$, appear but these are not relevant to the dominant repulsivecore singularity.) As evident from Fig. 3, the bifurcation point is located by the tangent passing through $\psi=-\beta U_{0}<0$ : this leads to

$$
0 \leq \psi_{0}(T)=\frac{1}{2} \beta U_{0}\left\{\left[1+\left(4 k_{B} T / U_{0}\right)\right]^{1 / 2}-1\right\}<1,
$$

and

$$
\begin{aligned}
z_{0}(T) & =-\left[1-\psi_{0}(T)\right] \exp \left[-\psi_{0}(T)\right], \\
& \approx-1 / e \beta U_{0}\left[1+\mathcal{O}\left(1 / \beta U_{0}\right)\right],
\end{aligned}
$$

the last result applying for strong repulsions $\left(\beta U_{0} \rightarrow\right.$ $\infty)$. Clearly, this saddle-point bifurcation represents the repulsive-core singularity.

Incidentally, if following Hauge and Hemmer [15], we had treated the $(d=1)$-dimensional continuum hard-rod gas with additional infinite-range, infinitely weak repulsive Kac potentials, we would, at this point, have found $z_{0}=-1 / e \beta U_{0}$ in precise accord with the exact (limiting) calculations 15. But, of course, our saddle-point treatment is not restricted to $d=1$ even though in the Kac limit it will also become exact.

To complete the analysis we may now follow standard procedures by expanding about the saddle point values $\varphi_{0}$ and $\chi_{0}$ [following from (15)-(17)]. In terms of $\delta \varphi_{j}=\varphi_{j}-\varphi_{0}, \delta \chi_{j}=\chi_{j}-\chi_{0}$, and $\delta \psi_{j}=\delta \chi_{j}-i \delta \varphi_{j}$, the Hamiltonian truncated at fourth order becomes

$$
\begin{aligned}
\mathcal{H}[\delta \varphi, \delta \chi]= & \frac{1}{2} \sum_{j, k}\left(\varphi_{j} w_{j k}^{-1} \varphi_{k}+\chi_{j} v_{j k}^{-1} \chi_{k}\right) \\
& +\sum_{j}\left[\frac{1}{2} r_{0}\left(\delta \psi_{j}\right)^{2}-g_{0}\left(\delta \psi_{j}\right)^{3}+u_{0}\left(\delta \psi_{j}\right)^{4}\right],
\end{aligned}
$$

where it is convenient to put

$$
\zeta_{l}(T, z) \equiv-z^{-1} e^{-\psi_{0}} /\left(1+z^{-1} e^{-\psi_{0}}\right)^{l},
$$

(which is real and positive for $z \simeq z_{0}$ when $l=0,2,4, \cdots$ ) so that $r_{0}=\zeta_{2}(T, z)$ and

$$
g_{0}=\frac{1}{6} \zeta_{3}\left(1+\zeta_{0}\right), \quad u_{0}=\frac{1}{24} \zeta_{4}\left(1+4 \zeta_{0}+\zeta_{0}^{2}\right) .
$$

In the usual continuum approximation this becomes

$$
\begin{gathered}
\mathcal{H}=\int d^{d} x\left[\frac{1}{2} r_{11} \delta \varphi^{2}(\mathbf{x})+r_{12} \delta \varphi(\mathbf{x}) \delta \chi(\mathbf{x})+\frac{1}{2} r_{22} \delta \chi^{2}(\mathbf{x})\right. \\
+\frac{1}{2} c_{\varphi}(\nabla \delta \varphi)^{2}+\frac{1}{2} c_{\chi}(\nabla \delta \chi)^{2} \\
\left.-g_{0} \delta \psi^{3}(\mathbf{x})+u_{0} \delta \psi^{4}(\mathbf{x})\right],
\end{gathered}
$$

with $c_{\varphi}=a^{2} / \beta \hat{w}_{0}$ and $c_{\chi}=b^{2} / \beta \hat{v}_{0}$ and

$$
\begin{aligned}
& r_{11}=k_{B} T / \hat{w}_{0}-\zeta_{2}(z, T), \\
& r_{22}=k_{B} T / \hat{v}_{0}+\zeta_{2}(z, T), \\
& r_{12}=-i \zeta_{2}(z, T) .
\end{aligned}
$$

Before proceeding generally, let us suppose that only repulsive terms act, i.e., $\hat{v}_{0}=0$. Then we may drop the integrals $\int \mathcal{D} \chi$ and see, by (17), that $\delta \psi=-i \delta \varphi$; but note that $\psi_{0}(T)$ remains real, positive and less than unity. The continuum Hamiltonian then reduces to

$$
\mathcal{H}_{0}[\delta \varphi]=\int d^{d} x\left[\frac{1}{2} t_{0}(\delta \varphi)^{2}+\frac{1}{2} c_{\varphi}(\nabla \delta \varphi)^{2}-i g_{0} \delta \varphi^{3}+u_{0} \delta \varphi^{4}\right]
$$

where the controlling coefficient is

$$
t_{0}(T, z)=r_{11}=\frac{1}{\beta \hat{w}_{0}}\left(1-\frac{z}{z_{0}}\right) \frac{\left[1-z z_{0} e^{2 \psi_{0}(T)}\right]}{\left[1+z e^{\psi_{0}(T)}\right]^{2}},
$$

which is positive for small $z$, decreases as $z=-|z|$ increases in magnitude, and vanishes linearly when $z \rightarrow$ $z_{0}(T)+<0$. The fourth order coefficient, $u_{0}$, is positive and approaches

$$
u_{00}=\frac{1}{4}\left(1-\psi_{0}\right)\left(1-\psi_{0}+\frac{1}{6} \psi_{0}^{2}\right) / \psi_{0}^{4}>0,
$$

when $z \rightarrow z_{0}(T)$, while the cubic term is purely imaginary with a coefficient approaching

$$
g_{00}=-\frac{1}{6}\left(1-\psi_{0}\right)\left(2-\psi_{0}\right) / \psi_{0}^{3}<0 .
$$


At this point we have thus reached the same stage as in the original treatment [1] of the Yang-Lee edge singularities. The imaginary term $i(\delta \varphi)^{3}$ dominates the behavior. A momentum-shell RG analysis reveals an upper critical dimension $d_{c}=6$, an exponent $\eta \approx-\frac{1}{9} \epsilon$ for $\epsilon=6-d>0$, and, using a unit cutoff in momentum space, a fixed point value of $g_{0}^{*} \approx\left(\epsilon / 54 K_{6}\right)^{1 / 2}$, where $K_{6}$ is the area of the unit sphere at $d=6$ dimensions. All other results follow as previously $[4,7]$.

Returning now to the general case of (25), in which attractive interactions are present (i.e., $\hat{v}_{0}>0$ ) but $U_{0}$ remains positive, we anticipate that only one linear combination of $\delta \varphi$ and $\delta \chi$ will become critical. To check this at the saddle-point level, it suffices to put

$$
\delta \chi(\mathbf{x})=\delta \chi^{\prime}(\mathbf{x})+i\left(\zeta_{2} / r_{22}\right) \delta \varphi(\mathbf{x}) .
$$

The quadratic (nongradient) terms in (25) then become

$$
\frac{1}{2} t(\delta \phi)^{2}+\frac{1}{2} r_{22}\left(\delta \chi^{\prime}\right)^{2}
$$

The coefficient $r_{22}(T, z)$ remains positive for all negative $z$ but one finds

$$
t(T, z)=r_{11}-\frac{r_{12}^{2}}{r_{22}} \approx \frac{t_{0}(T, z)}{\left[1+\beta \hat{v}_{0}\left(1-\psi_{0}\right) / \psi_{0}^{2}\right]}
$$

for $z \simeq z_{0}$ : thus by (28), $t$ vanishes when $z \rightarrow z_{0}(T)$. Integration over the noncritical field $\delta \chi^{\prime}(\mathbf{x})$ is hence justified and may be carried out perturbatively. Apart from additive terms that are nonsingular near $z_{0}(T)$, this finally leads to a renormalized LGW Hamiltonian

$$
\begin{aligned}
\mathcal{H}_{R}[\delta \varphi]=\int d^{d} x & {\left[\frac{1}{2} t_{R}(\delta \varphi)^{2}+\frac{1}{2} a_{R}^{2}(\nabla \delta \varphi)^{2}\right.} \\
& \left.-i h_{R} \delta \varphi-i g_{R}(\delta \varphi)^{3}+\cdots\right]
\end{aligned}
$$

where the fourth and higher order terms have been dropped. For $\hat{v}_{0}$ sufficiently small, the renormalized couplings $t_{R}$ and $g_{R}$ will differ little from $t$ and $g$ and, in particular, $h_{R}$ and $g_{R}$ will be real, so that the $i(\delta \varphi)^{3}$ term once again dominates.

Stability of the saddle point requires that the renormalized repulsive range, $a_{R}$, in (34) be real (and positive). Roughly, we expect $a_{R}^{2} \propto\left(a^{2} \hat{w}_{0}-b^{2} \hat{v}_{0}\right)$ : the positivity of this factor then represents a previously unstated restriction on the initial potentials. In practice, however, if this (or the more precise) condition fails, it may be sufficient, as explained in the discussion of gas-liquid criticality, to perform further, nonperturbative renormalizations to dampen the attractive interactions for $z<0$ and so expose again a saddle-point representation of the repulsive-core singularity at $z_{0}(T)$. Certainly, we must expect the form (34) to represent the singularity whenever it is actually realized in a system; and, then, clearly it must belong to the Yang-Lee edge universality class.

\section{CONCLUSIONS}

In summary, by using separate field-theoretic transformations for the repulsive and attractive parts of the pair interactions in a fluid, we have demonstrated in general the presence of a universal repulsive-core singularity on the negative axis at a value $z_{0}(T)<0$ : see Fig. 1 . The behavior of the pressure in the vicinity of $z_{0}$ is stated in (6). The singularity belongs to the same universality class as Yang-Lee edge criticality, as proposed by Lai and Fisher [7]: see Eqs. (1)-(4) and Fig. 1. The basic critical exponents are related via (7) (see also [7]); the borderline dimensionality is $d_{c}=6$; and an $i \varphi^{3}$ coupling characterizes the LGW fixed-point Hamiltonian [4].

\section{ACKNOWLEDGMENTS}

Y. P thanks the Institute for Physical Science and Technology at the University of Maryland for hospitality. Her work has been supported in part by the Ministry of Education of Korea through the Basic Science Research Institute of Seoul National University. M.E.F. acknowledges support from the U. S. National Science Foundation (through Grant CHE 96-14495). The interest of Youngchan Kim has been appreciated.

[1] T. D. Lee and C. N. Yang, Phys. Rev. 87, 410 (1952).

[2] C. N. Yang and T. D. Lee, Phys. Rev. 87, 404 (1952).

[3] P. J. Kortman and R. B. Griffiths, Phys. Rev. Lett. 27, 1439 (1971).

[4] M. E. Fisher, Phys. Rev. Lett. 40, 1610 (1978).

[5] O. F. De Alcantara Bonfim, J. E. Kirkham, and A. J. McKane, J. Phys. A 13, L247 (1980).

[6] M. E. Fisher, Prog. Theoret. Phys. Suppl. 69, 14 (1980).

[7] S.-N. Lai and M. E. Fisher, J. Chem. Phys. 103, 8144 (1995).

[8] G. Parisi and N. Sourlas, Phys. Rev. Lett. 49, 871 (1981).

[9] A. R. Day and T. C. Lubensky, J. Phys. A 15, L285 (1982).

[10] T. C. Lubensky and A. J. McKane, J. Phys. Lett. (Paris) 42, L331 (1981).

[11] J. L. Cardy, J. Phys. A 15, L593 (1982).

[12] J. Groenevold, Phys. Lett. 3 , 50 (1962).

[13] D. Poland, J. Stat. Phys. 35, 341 (1984).

[14] A. Baram and M. Luban, Phys. Rev. A 36, 760 (1987).

[15] E. Hiis Hauge and P. C. Hemmer, Physica 29, 1338 (1963).

[16] R. J. Baxter, J. Phys. A 13, L61 (1980) has obtained exact results for the hard-hexagon lattice gas.

[17] D. A. Kurtze and M. E. Fisher, Phys. Rev. B 20, 2785 (1979).

[18] Y. Shapir, J. Phys. A 15, L433 (1982). 
[19] J. Glimm and A. Jaffe, Quantum Physics: A Functional Point of View, 2nd ed. (Springer Verlag, Berlin, 1987).

[20] M. Kac, Phys. Fluids 2, 8 (1959).

[21] J. Hubbard, Phys. Rev. Lett. 3, 77 (1954).

[22] R. L. Stratonovich, Doklady Akad. Nauk SSSR 115, 1097 (1957) [Sov. Phys. Doklady 2, 416 (1957)].

[23] A. G. Moreira, M. M. Telo da Gama, and M. E. Fisher, J. Chem. Phys. 110, 10058 (1999).

[24] R. R. Netz and H. Orland, One and two-component hardcore plasmas (cond-mat/9902220, 16 Feb 1999).

[25] J. W. Negele and H. Orland, Quantum Many Particle Systems (Addison Wesley, 1988) p. 199.

[26] J.Hubbard and P. Schofield, Phys. Lett. 40A, 245 (1972).

[27] N. V. Brilliantov, Phys. Rev. E 58, 2628 (1998).

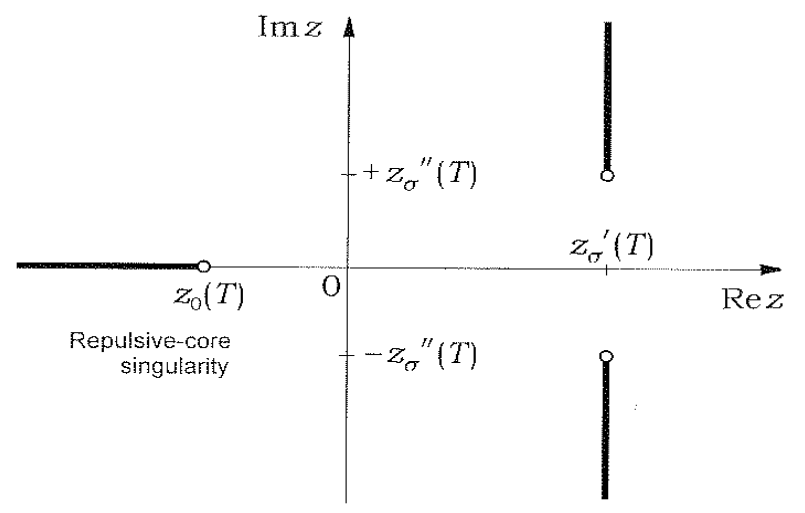

FIG. 1. Complex activity plane for a lattice or continuum fluid system at temperatures $T>T_{c}$, above gas-liquid criticality. The Yang-Lee edge singularities are located at $z=z_{\sigma}^{\prime}(T) \pm i z_{\sigma}^{\prime \prime}(T)$ while the repulsive-core singularity lies on the real negative activity axis $z=z_{0}(T)$. (Note that the branch cuts running from the Yang-Lee edges will not, in general, be linear as shown, purely schematically, here: indeed, for a simple lattice-gas the cuts lie on circles centered at $z=0$.)

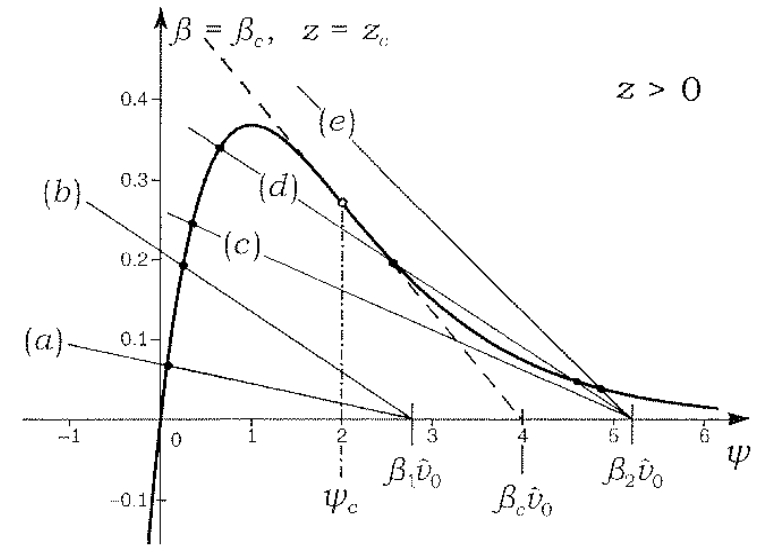

FIG. 2. Plot of $\psi e^{-\psi}$ (bold curve) versus $\psi$ to elucidate solutions of the saddle-point equation (18) in the case of only single-site repulsive hardcores with attractive potentials of strength $\hat{v}_{0}\left(=-U_{0}\right)$ for various temperatures, $\beta_{1}, \beta_{c}$ and $\beta_{2}$ and positive activities $z$. The open circle marks the point of inflection which serves to locate the (classical) gas-liquid critical point; the dashed line is the associated tangent. The solution lines have slopes $-z$ in accord with (18).

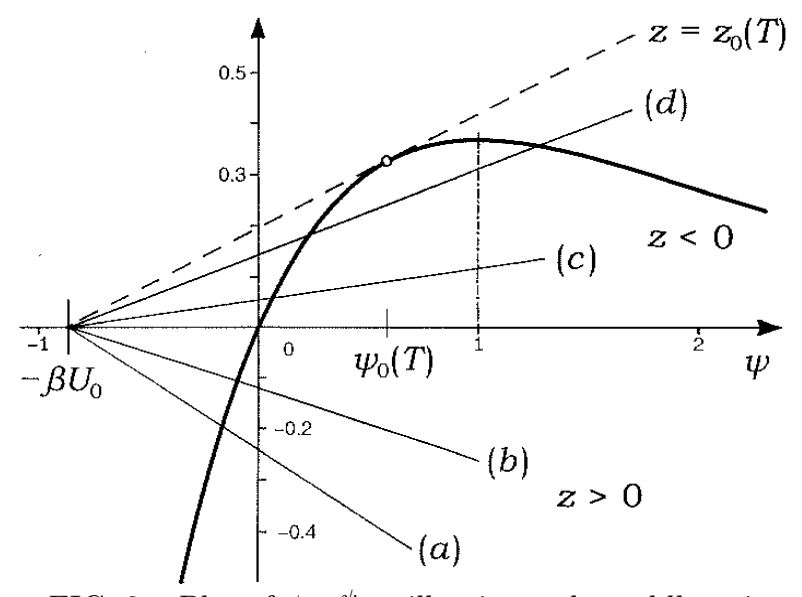

FIG. 3. Plot of $\psi e^{-\psi}$ to illuminate the saddle-point equation (18) as in Fig.2; but note the change in scales. The dominant repulsive case with $U_{0}=\hat{w}_{0}-\hat{v}_{0}>0$ is illustrated for positive activities $z$ [lines (a) and (b)] and negative activities: (c) and (d) and the tangent (dashed line) which locates the repulsive core-singularity at $z=z_{0}(T)<0$ with field $\psi_{0}(T)$. 